\title{
Max-Min Ant System based Approach for Intelligent VM Migration and Consolidation for Green Cloud Computing
}

\author{
Reena Sarathe \\ TIT Bhopal
}

\author{
Amit Mishra \\ TIT Bhopal
}

\author{
Shiv Kumar Sahu, PhD \\ TIT Bhopal
}

\begin{abstract}
Cloud computing has bring a revolution in the field of computing. Many algorithms are proposed to make it even more efficient. In cloud computing Virtualization plays an important role and whole performance of cloud depends on VM allocation and Migration. As lots of energy is consumed in this technology so algorithms to save energy and improve efficiency are proposed called Green algorithms. In this paper a green algorithm for VM Migration is proposed using metaheuristic algorithm called ACO. The variant of ACO used in this paper is Max-Min Ant System. Results show that MaxMin Ant System gives best result as compared to other approaches in terms of VM Migrations, VM consolidation and energy consumptions.
\end{abstract}

\section{Keywords}

Meta-heuristic, Max-Min Ant System, Virtual Machine (VM).

\section{INTRODUCTION}

Cloud computing is defined as the ability to use computing resources - applications, storage and processing power over the internet. These computing resources are hosted and managed by "someone else" (the cloud provider). It is an approach to maximize the capacity or step up capabilities without investing in new infrastructure. It provides gigantic storage for data storage and high performance computing to customers over the web [3]. Cloud primarily refers to saving of user's data to an offsite storage system that is maintained by a third party. This means instead of storing information on user computer's hard disk or other storage devices, client save it to a remote database where internet provides the connection between user computer and the remote database [3].Clouds enable platform for dynamic and flexible application provisioning by exposing data center's capabilities as a network of virtual services. So users can access and deploy applications from anywhere in the Internet driven by demand and QoS requirements.

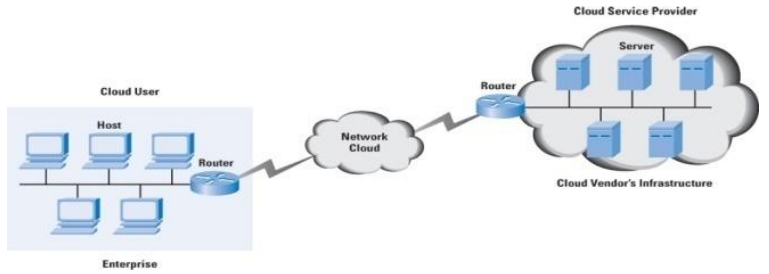

Fig 1.1: Figure showing cloud

\section{Cloud Computing Services:}

Cloud computing service models or offerings can be classified into 3 segments :

-IaaS - Infrastructure as a Service: Includes servers, storage, virtual machines, load balancers and other core infrastructure stack. It is used by large sized organization [4].Here we need to configure Server through Network Administrator . For develop and Deploy software we need skilled IT developer team.Leading IaaS Service Provider are Amazon, Rackspace, IBM and HP.

-PaaS (Infrastructure + Platform)- Platform as a Service. Adds development and programming models to IaaS. Includes databases, execution frameworks/runtimes, web servers and development tools. Here we need only Programmers team to develop and deploy software [4].

Leading Paas Service Provider are Google app Engine, Windows azure, force.com.

-SaaS(Infrastructure + Platform +Software)- Software as a Service. Complete application offering in the cloud [4]. Salesforce CRM, Google Apps/Gmail/google drive/gtalk/GoogleCalendar, Microsoft "Live", Dropbox, and a lot more. Here we only select a software that is offered by service providers, and we customize a software as our requirement. Need only Administrator or Cloud Consultant for initial setup.

\section{LITERATURE REVIEW}

In [12] an admission control and scheduling mechanism proposes which not only maximizes the resource utilization and profit, but also ensures that the QoS requirements is proposed. Mixed Workload Aware Policy (MWAP) is implemented to consider the workload of different types of application such transactional and non-interactive batch jobs. The proposed mechanism provides substantial improvement over static server consolidation and reduces SLA violations. In [13] VM consolidation problem which is a NP Hard problem is solved by applying meta-heuristic algorithm ACO. The objective is to lower down the energy consumption of the overall algorithm. And the algorithm also reduces VM migrations. In [6] a novel allocation and selection policy for the dynamic virtual machine (VM) consolidation in virtualized data centers to reduce energy consumption and SLA violation. Firstly, it detect overloading hosts in virtual environments and then apply a method to select VMs from those overloading hosts for migration. VM Provisioning Method to Improve the Profit and SLA Violation of Cloud Service Providers. In [2] authors proposed an Threshold based algorithm for VM provisioning among multiple service providers that reduces SLA Violation.

It uses two threshold values and two type of VMs (on-demand and reserved),These threshold values will be decided by the cloud federation depending on the environmental conditions like current workload, idle capacity of each cloud provider, etc. In [11] a power friendly algorithm is proposed. This paper compared live and non live VM migration in terms of power consumption.

In [16] authors developed an objective method to facilitate the comparison of different virtual machine placement algorithms in the cloud. In [17]stable matching framework to decouple policies from mechanisms when mapping virtual machines to physical servers are presented and a general resource management architecture called Anchor is proposed. In [18] the resource allocation problem to be a convex optimization 
problem and proposed a self-organizing cloud architecture is discussed. Speitkamp and Bichler [20] studied the static consolidation problem with a mathematical programming approach.In [19] they modelled the consolidation as a modified bin-packing problem. These works focus on the initial VM deployment or static consolidation problem based on resource utilization and do not consider VM migration overhead.

\section{ANT SYSTEM}

Consider each physical machine is represented by a node in graph and each edge defines VM migration from one physical machine (PM) to another. The generated graph will be directed and completely connected having positive edge weights.

Consider 3 physical machines having one. Two, three VMs respectively.

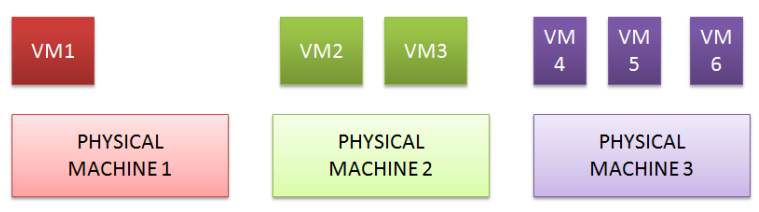

Fig 1.2: Figure showing three physical machines and corresponding VMs

The above situation can be converted into graph as shown below.

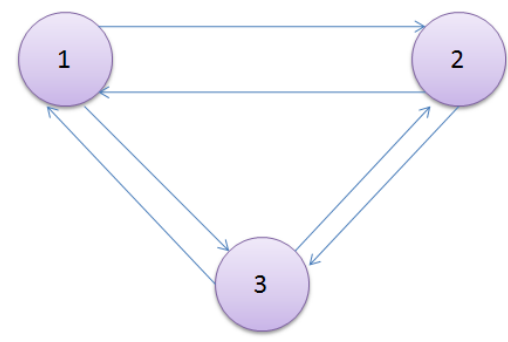

Fig 1.3: Graph representing PMs and edges represent VM migration probability.

In AS ants concurrently build the solution for the Cloud VM. Initially ants are put on randomly chosen nodes which represent PM. In each iteration construction step, ant $\mathrm{k}$ applies probabilistic action choice rule, called random proportional rule, to decide to which PM given VM should be migrated. $\mathrm{P}_{\mathrm{ij}}$ is the probability of migrating VM to $j$ which is currently at $i$ is:

$$
p_{i j}^{k}=\frac{\left[\tau_{i j}\right]^{\alpha}\left[\eta_{i j}\right]^{\beta}}{\sum_{l \in N_{i}^{k}}\left[\tau_{i j}\right]^{\alpha}\left[\eta_{i j}\right]^{\beta}} \quad \text { if } j \in N_{i}^{k}
$$

Where $\eta_{\mathrm{ij}}=1 / \mathrm{d}_{\mathrm{ij}}$ is a heuristic. $\alpha$ and $\beta$ are two parameters which determine the relative influence of the pheromone trail and the heuristic information, and where $N_{i}^{k}$ is the nodes which are available.

\section{Update of Pheromone Trail}

When ants have constructed their tours, pheromone trails are updated. This is done by first lowering the pheromone value on all the edges by a constant factor called $\rho$. And then adding the pheromone content on the edges visited by the ants in their tours. Pheromone evaporation is done as:

$$
\tau_{i j} \leftarrow(1-\rho) \tau_{i j} \quad, \text { for all }(i, j) \epsilon L .
$$

Where $\rho$ is the pheromone evaporation rate lies between $0<\rho<=1$. The parameter $\rho$ is used to avoid unlimited pheromone deposition and it enables the algorithm to not to choose the bad path that were chosen by earlier ants. The pheromone evaporation decreases the pheromone content exponentially with the number of iterations. In first iteration pheromone is $\rho$ times the initial pheromone available but in second iteration pheromone available is $\rho^{2}$ times the pheromone available initially. After evaporation, all ants deposit pheromone in their tour constructed in tour construction phase. Pheromone is updated as:

$$
\tau_{i j} \leftarrow \tau_{i j}+\Delta \tau_{i j}^{i}
$$

Where $\Delta \tau_{i j}^{i}$ is the amount of pheromone deposited by ant $\mathrm{k}$ on the arcs it has visited. It is defined as:

$$
\begin{aligned}
& \Delta \tau_{i j}^{i}= \\
& \left\{\begin{array}{cc}
1 / n^{k} & \text { if } \operatorname{arc}(i, j) b e l o n g s \text { to the tour of the ant } k \\
0 & \text { otherwise }
\end{array}\right.
\end{aligned}
$$

Where $n^{k}$ is the number of VMs running on kth PM. With this less the number of VMs on PM more is the amount of the pheromone deposit on the edges of its tour. Therefore more likely they will be chosen by ants in their future iterations of the algorithm and relatively less difference in pheromone trail levels.

\subsection{Max-Min Ant System}

In Max-Min Ant System, after all the ants of single iteration have completed the tour than pheromone is evaporated with the same concept as the pheromone is evaporated in Ant system. Now new pheromone is deposited as follows:

$$
\tau_{i j} \leftarrow \tau_{i j}+\Delta \tau_{i j}^{\text {best }}
$$

Where $\Delta \tau_{i j}^{\text {best }}=1 / \mathrm{n}^{\text {kbest }}$. The ant which is allowed to add pheromone may be either the best-so-far, or the iteration-best.

\subsection{Experimental Setup}

In this paper cloudSim is used to simulate cloud environment. CloudSim is a java based library for simulating cloud.

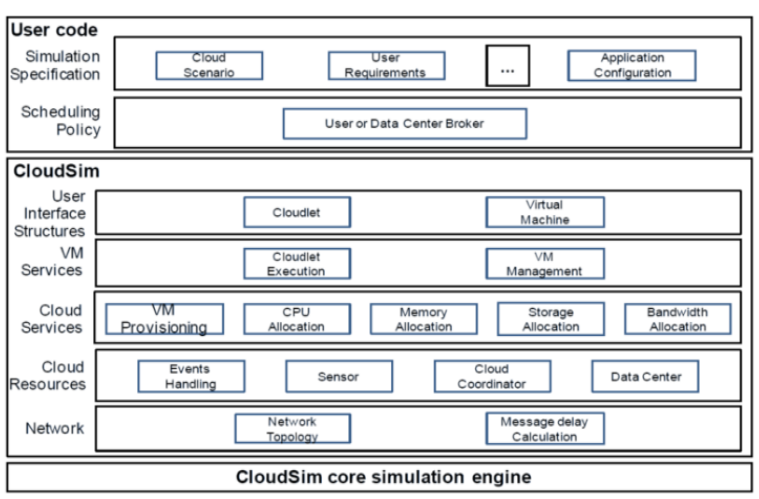

Fig 1.4: Figure showing CloudSim architecture

\section{CloudSim steps for Simulation:}

- $\quad$ Set the no. of user.

- Initialization of common variable.

- CIS will be created by using init method. 
- Datacenter will be created by using createDatacenter method. In this for each datacenter ,we create a host with its characteristics.

- Datacenter broker instance will be created

- Create Instance of virtual machine with PE ,RAM and Bandwidth requirement.

Now this virtual machine is submitted to broker.

Infrastructure has been developed at this point.

- Cloudlet is created with Bandwidth and MIPS requirement.

- Now this Cloudlet will get submitted to Broker.

- Start Simulation process

- Stop Simulation process

- $\quad$ Print the status of the Simulation.

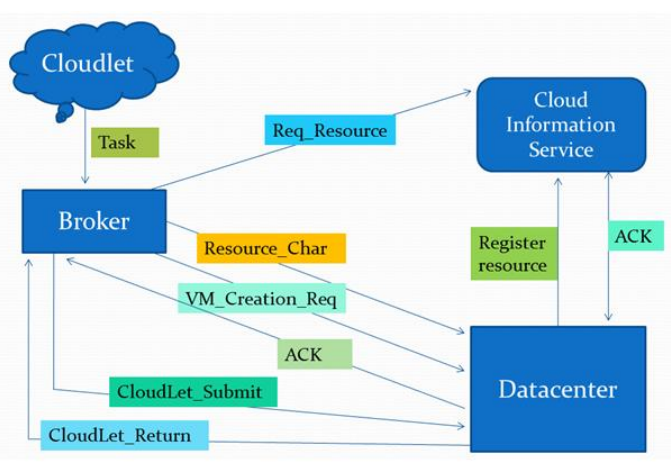

Fig 1.5: Figure showing cloud architecture.

\section{RESULTS}

In this paper a cloud is simulated using cloudsim having fixed number of physical machines and virtual machines. Configuration of physical and virtual machi.nes is measured in terms of MIPS (million instructions per second). VM Migrations, VM consolidation and energy consumption are recorded.

Physical machines are created based on the following MIPS list as shown below:

$\{750,1000,1500,2000\}$

Virtual machines are created based on the following MIPS list as shown below:

\section{$\{250,500,750,1000\}$}

No. of VM Migrations are compared for traditional VM Migration approach (TVMM), ACO based approach (ACOVMM) and Max-Min based approach (MMVMM).

Table 1.1: Table showing no. of VM migrations for different approaches

\begin{tabular}{|c|c|c|c|c|}
\hline \multicolumn{5}{|c|}{ No. of VM Migrations } \\
\hline $\begin{array}{c}\text { No. of } \\
\text { PMs }\end{array}$ & $\begin{array}{c}\text { No. of } \\
\text { VMs }\end{array}$ & TVMM & ACOVMM & MMVMM \\
\hline 10 & 15 & 7 & 4 & 1 \\
\hline 15 & 20 & 9 & 6 & 2 \\
\hline 20 & 25 & 11 & 9 & 6 \\
\hline 25 & 30 & 16 & 13 & 10 \\
\hline 30 & 35 & 19 & 15 & 11 \\
\hline
\end{tabular}

Table 1.2: Table showing no. of VM consolidations for different approaches

\begin{tabular}{|c|c|c|c|c|}
\hline \multicolumn{5}{|c|}{ No. of VM Consolidation } \\
\hline $\begin{array}{c}\text { No. of } \\
\text { PMs }\end{array}$ & $\begin{array}{c}\text { No. of } \\
\text { VMs }\end{array}$ & TVMM & ACOVMM & MMVMM \\
\hline 10 & 15 & 2 & 5 & 7 \\
\hline 15 & 20 & 3 & 3 & 6 \\
\hline 20 & 25 & 5 & 7 & 10 \\
\hline 25 & 30 & 8 & 11 & 14 \\
\hline 30 & 35 & 11 & 13 & 18 \\
\hline
\end{tabular}

Table 1.3: Table showing energy consumption for different approaches

\begin{tabular}{|l|l|l|l|l|}
\hline \multicolumn{5}{|c|}{ Energy Consumption in KWH } \\
\hline $\begin{array}{l}\text { No. of } \\
\text { PMs }\end{array}$ & $\begin{array}{l}\text { No. of } \\
\text { VMs }\end{array}$ & TVMM & ACOVMM & MMVMM \\
\hline 10 & 15 & 3 & 2 & 1 \\
\hline 15 & 20 & 5 & 3 & 2 \\
\hline 20 & 25 & 7 & 6 & 6 \\
\hline 25 & 30 & 8 & 8 & 7 \\
\hline 30 & 35 & 12 & 11 & 10 \\
\hline
\end{tabular}

5. CONCLUSION AND FUTURE WORK

In this paper an Ant colony approach is proposed for VM migration in cloud. As described in this paper VM Migration is a NP-Hard problem and this problem can be solved in less time using some meta-heuristic algorithm. All such implementations can be effectively simulated using a tool called CloudSim. And ACO has many variants; different variants may be applied on VM migration problem. In this paper Max-Min ant system is applied and Performance of all these variants is compared with each other in terms of No. of VM Migrations, Energy consumption and VM consolidation. It is concluded that Max-Min approach gives best results

\section{REFERENCES}

[1] Komal Singh Patel and A. K. Sarje, "VM Provisioning Method to Improve the Profit and SLA Violation of Cloud Service Providers," IEEE International Conference, Cloud Computing in Emerging Markets (CCEM) 11-12 Oct. 2012.

[2] K. S. Patel and A.K. Sarje, "VM Provisioning Policies to Improve the Profit of Cloud Infrastructure Service Providers," ICCCNT-12, July.2012.

[3] Gundeep Singh Bindra, Prashant Kumar Singh,Seema Khanna,Krishen Kant Kandwal, "Cloud Security : Analysis and Risk Management of VM Images," Proceeding of IEEE International Conference on Information and Automation Shenyang, China, June 2012.

[4] Eeraj Jan Qaisar, "Introduction to Cloud Computing for Developers,” In IEEE C2012.

[5] A. Beloglazov, R. Buyya, "Optimal Online Deterministic Algorithms and Adaptive Heuristics for Energy and Performance Efficient Dynamic Consolidation of Virtual Machines in Cloud Data Centers," Concurrency and Computation: Practice and Experience (CCPE), Wiley Press, New York, USA, Sep. 2012, pp. 1397-1420, doi: 10.1002/cpe.1867.

[6] Zhibo Cao and ShoubinDong, "Dynamic VM consolidation for energy-aware and SLA violation reduction in cloud computing," 13th International 
Conference on Parallel and Distributed Computing, Applications and Technologies 2012.

[7] YonggenGu, Wei Zhang, YonggenGu, Jie Tao, “A Study of SLA Violation Compensation Mechanism in Complex Cloud Computing Environment,” In IEEE (C) 2012.

[8] C. Belady, "In the data center, power and cooling costs more than the equipment it supports," 2007. URL http://www.electronicscooling.com/articles/2007/feb/a3/.

[9] http://en.wikipedia.org.

[10] http://www.sciencedirect.com/science/article/pii/S18777 05811054117.

[11] David Aikema, AndreyMirtchovski, Cameron Kiddle, and Rob Simmonds "Green Cloud VM Migration: Power Use Analysis" in IEEE 2012.

[12] Saurabh Kumar Garg, Adel NadjaranToosi, Srinivasa K. Gopalaiyengar, RajkumarBuyya, "SLA-based virtual machine management for heterogeneous workloads in a cloud datacenter," Journal of Network and Computer Applications 1 August 2014.

[13] Rafid Sagban, Ku Ruhana Ku Mahamud, Muhamad Shahbani Abu Bakar "Reactive Memory Model for Ant Colony Optimization and Its Application to TSP" in 2014 IEEE International Conference on Control System, Computing and Engineering, 28 - 30 November 2014, Penang, Malaysia.
[14] M. Veluscek, T. Kalganova, P. Broomhead "Improving Ant Colony Optimization Performance through Prediction of Best Termination Condition" in IEEE 2015.

[15] Fahimeh Farahnakian, Adnan Ashraf, TapioPahikkala,PasiLiljeberg, JuhaPlosila, Ivan Porres, and HannuTenhunen "Using Ant Colony System to ConsolidateVMs for Green Cloud Computing" in IEEE TRANSACTIONS ON SERVICES COMPUTING, VOL. 8, NO. 2, MARCH/APRIL 2015.

[16] K. Mills, J. Filliben, and C. Dabrowski, "Comparing vmplacement algorithms for on-demand clouds," in Proc. IEEE 3rd Int. Conf. Cloud Comput. Tech. Sci., 2011, pp. 91-98.

[17] H. Xu and B. Li, "Anchor: A versatile and efficient framework for resource management in the cloud," IEEE Trans. Parallel Distrib. Syst., vol. 24, no. 6, pp. 1066 1076, Jun. 2013.

[18] S. Di and C.-L. Wang, "Dynamic optimization of multiattribute resource allocation in self-organizing clouds," IEEE Trans. Parallel Distrib. Syst., vol. 24, no. 3, pp. 464-478, Mar. 2013.

[19] S. Srikantaiah, A. Kansal, and F. Zhao, "Energy aware consolidation for cloud computing," in Proc. Conf Power Aware Comp. Syst., 2008, pp. 10-10.

[20] B. Speitkamp and M. Bichler, "A mathematical programming approach for server consolidation problems in virtualized data centers," IEEE Trans. Serv. Comput., vol. 3, no. 4, pp. 266-278, Oct. 2010. 